\title{
The Relationship between College Students' Critical Behavior and the Coping Style in China
}

\author{
Qing Zeng \\ Mental Health Center, Jinan University, Guangzhou, China \\ Email:330659812@qq.com
}

How to cite this paper: Zeng, Q. (2017). The Relationship between College Students' Critical Behavior and the Coping Style in China. Psychology, 8, 2390-2397. https://doi.org/10.4236/psych.2017.814150

Received: November 13, 2017 Accepted: December 10, 2017 Published: December 13, 2017

Copyright (C) 2017 by author and Scientific Research Publishing Inc. This work is licensed under the Creative Commons Attribution International License (CC BY 4.0).

http://creativecommons.org/licenses/by/4.0/

\begin{abstract}
This study aims to study the relationship between college students' critical behavior and the coping style in China. 1135 college students from 10 universities in Guangdong were asked to answer the questionnaire on the behaving style in emergency situations and Coping Style questionnaire. The results show that: 1) In the view of gender, admission category, urban and rural sources, whether the only child, and family income point, there were significant differences in the behaviors in emergency situations. 2) The capacities in emergency situations and the coping style are significantly correlative. Meanwhile, the coping styles of self-blame, problem solving, fantasy, rationalization are the distinct predictors for the capacities in emergency situations.
\end{abstract}

\section{Keywords}

College Students, Critical Behavior, Coping Style

\section{Introduction}

The students' behavior patterns are mainly divided into two cases (Jex \& Bliese, 1999, 2001): a deal with critical incidents of behavior and critical situational behavior. Critical situation is taken when people unexpectedly encounter some unexpected danger or face some kinds of things, which is in danger, or affects personal safety of themselves or others. Critical situations will cause a sudden event behavior; the other is personal coping styles. Researchers have studied the relationship of the critical situational behavior with coping styles. However subjects are mainly concentrated in the military academies or the officers and the armymen (Li \& Zhang, 2004; Ning \& Li, 2012; Dang \& Yang, 2012). The results showed that the positive acute psychological stress reaction behavior and the positive coping style were significantly related, and the negative acute psycho- 
logical stress reaction behavior which would shock response behavior and the negative coping style were significantly related. But the researches were mainly about acute stress (such as the war situation) and chronic stress (such as post-traumatic stress). The first aim of the current study is to research how college students deal with the sudden situational changes or life events, and the related coping styles. The second aim is to research the relationship of the two behaviors.

\section{Methods}

\subsection{Participants}

1200 freshman and sophomore students of 10 Universities in Guangdong Province in China volunteered participant this questionnaire, and recovered 1189 copies, of which 1135 were valid questionnaires. The remaining sample of participants (mean age $=19.5$ years, $\mathrm{SD}=2.6$ ) consisted of 539 men $(47.5 \%)$; the Enrollment category, within enrollment (referring to students from mainland China) are 1069 (94.2\%), outside enrollment (referring to mainland China outside students, including Hong Kong, Macao, Taiwan, overseas Chinese, foreign students) 66 (5.8\%); and the only child 397 (35.0\%); 593 people from the towns (52.2\%), 492 in rural areas (43.3\%), missing 50 (4.4\%); 16 people from high-income families (1.4\%), 729 people from income families (64.2\%), and 387 people from low-income families (34.1\%), missing 3 people $(0.3 \%)$. The questionnaire was approved by the Academic Committee of the University.

\subsection{Measures}

Students critical situational behavior questionnaire (CSB). Reference the questionnaires of "Stressor Scale", "Stressful Life Events Scale" and "Crisis Handling Tests" (Liang \& Hao, 2005). Through individual interviews and open questionnaires, we collected the coping style and the possible critic situational behavioral patterns which include university students encountered critical situation, for example when facing the death of a loved one, traffic accident, economic distress, be damaged self-esteem. After screening merge, we sorted out 35 questions. And we select another 195 students to finish the questionnaire, delete poor discriminated items, and finalize the formal critical situational behavior questionnaire of 30 questions, such as the question "If thugs armed with knives to rob you, what will you do" and the another question "Assuming the sudden death of one of the parents, how will you feel". Referenced to other similar scale, regardless of dimension, this questionnaire calculated the total score only. And the higher the score, the worse behavior in crisis situations. Measurements in the study, the internal consistency of the questionnaire coefficient $\alpha=0.61$. Using confirmatory factor analysis estimated that one-dimensional test reliability method confirmatory factor analysis (Ye \& Yang, 2011), model fit index RMSEA = $0.056, \mathrm{NNFI}=0.921, \mathrm{CFI}=0.931$, a good model fit. Also choose another 100 college students who had finished both students critical situational behavior 
questionnaire and crisis handling tests as a criterion, the average scores of the two are high related. The results show that the average scores of the two scales correlation coefficient of $0.76, \mathrm{p}<0.005$, which shows that students' critical situational behavior questionnaire is ideal.

Coping Style Questionnaire (Ye \& Yang, 2011). Total of 62 questions revised in Chinese by Jihua Xiao, which contains six subscales, such as problem-solving (PS), self-blame (SB), help, fantasy, retreat and rationalization (RL). Each scale has several questions as subscales, calculate the total score of each subscale, the higher the scores, and more that the use of the type of coping styles. Measurements in this study showed that part of the internal consistency coefficient $\alpha=0.69$.

\subsection{Procedure}

Make the group test way. Using the unified guidance language, participants in classes were told to fill out the survey, and the answer to the secret way.

\section{Results}

\subsection{College Students' Current Status of Critical Situational Behavior and Coping Styles}

From gender, enrollment category, different urban and rural sources, whether only one child, different family income levels that 5 aspects were compared. There was a significant difference in help and rationalization factors between boys and girls. Specifically, girls scored significantly higher than boys in the help factor, boys scored significantly higher than girls in the rationalization factor. There also was a significant difference in critical situational behavior between the inside and outside enrollment, the scores of inside enrollments students significantly lower than the outer enrollment. The data is showed in Table 1. And there were significant differences in critical situations, self-blame, avoidance factors between the urban and rural students. And in the three factors, the urban students scored significantly lower than students from rural areas. There were significant differences in help and retreat factors between the only child or not

Table 1. Mean (and SE) distribution of critical situational behavior and coping style scores based on gender and enrollment category.

\begin{tabular}{ccccccc}
\hline Factor & $\mathrm{M}$ & $\mathrm{F}$ & $\mathrm{t}$ & Inside & Outside & $\mathrm{t}$ \\
\hline & $(\mathrm{M} \pm \mathrm{SD})$ & $(\mathrm{M} \pm \mathrm{SD})$ & & $(\mathrm{M} \pm \mathrm{SD})$ & $(\mathrm{M} \pm \mathrm{SD})$ & \\
CSB & $15.49 \pm 5.3$ & $15.98 \pm 5.00$ & -1.61 & $15.56 \pm 5.10$ & $17.15 \pm 5.83$ & $-2.29^{*}$ \\
PS & $0.76 \pm 0.17$ & $0.75 \pm 0.17$ & 0.15 & $0.76 \pm 0.17$ & $0.72 \pm 0.17$ & 1.81 \\
SB & $0.39 \pm 0.21$ & $0.39 \pm 0.20$ & 0.62 & $0.38 \pm 0.21$ & $0.42 \pm 0.23$ & -1.32 \\
Help & $0.65 \pm 0.21$ & $0.72 \pm 0.19$ & $-5.87^{* *}$ & $0.69 \pm 0.20$ & $0.69 \pm 0.21$ & -0.06 \\
Fantasy & $0.45 \pm 0.20$ & $0.46 \pm 0.20$ & -1.38 & $0.46 \pm 0.20$ & $0.43 \pm 0.20$ & 0.88 \\
Retreat & $0.46 \pm 0.17$ & $0.46 \pm 0.17$ & -0.22 & $0.46 \pm 0.17$ & $0.48 \pm 0.17$ & -1.14 \\
RL & $0.42 \pm 0.18$ & $0.39 \pm 0.16$ & $3.16 * *$ & $0.40 \pm 0.17$ & $0.48 \pm 0.18$ & -4.02 \\
\hline
\end{tabular}


students, and the only child scored significant higher than non-only child. The data is showed in Table 2.

\subsection{Students' Behavior Patterns of Different Family Income Levels}

In critical situations, self-blame factor significantly different. Further multiple comparisons, critical situational factors on college students from high-income families score significantly higher than those from middle-income, low-income families of the students. In self-blame factor, college students from low-income families scored significantly higher than the college students from middle-income families. Help factor, college students from middle-income families score significantly higher than the college students from low-income families. The data is showed in Table 3.

\subsection{The Relationship of Critical Situational Behavior and Coping Styles}

As the capacity of critical situations questionnaire, higher the score, the lower

Table 2. Mean (and SE) distribution of critical situational behavior and coping style scores based on the point and the child.

\begin{tabular}{ccccccc}
\hline Factor & $\mathrm{M}$ & $\mathrm{F}$ & $\mathrm{t}$ & One-child & not one-child & $\mathrm{t}$ \\
\hline & $(\mathrm{M} \pm \mathrm{SD})$ & $(\mathrm{M} \pm \mathrm{SD})$ & & $(\mathrm{M} \pm \mathrm{SD})$ & $(\mathrm{M} \pm \mathrm{SD})$ & \\
\hline CSB & $15.21 \pm 4.83$ & $16.30 \pm 5.34$ & $-3.52^{\star *}$ & $15.61 \pm 4.72$ & $15.82 \pm 5.37$ & -0.65 \\
PS & $0.76 \pm 0.17$ & $0.75 \pm 0.17$ & 1.51 & $0.76 \pm 0.17$ & $0.75 \pm 0.17$ & 0.92 \\
SB & $0.37 \pm 0.20$ & $0.40 \pm 0.22$ & $-2.00^{*}$ & $0.37 \pm 0.19$ & $0.39 \pm 0.22$ & -1.46 \\
Help & $0.69 \pm 0.20$ & $0.69 \pm 0.20$ & 0.29 & $0.71 \pm 0.20$ & $0.68 \pm 0.20$ & $1.92^{\star}$ \\
Fantasy & $0.45 \pm 0.20$ & $0.46 \pm 0.21$ & -1.35 & $0.45 \pm 0.20$ & $0.46 \pm 0.20$ & -0.59 \\
Retreat & $0.44 \pm 0.17$ & $0.47 \pm 0.18$ & $-2.73^{* *}$ & $0.44 \pm 0.17$ & $0.46 \pm 0.17$ & $-1.98^{*}$ \\
RL & $0.40 \pm 0.17$ & $0.40 \pm 0.16$ & -0.45 & $0.40 \pm 0.17$ & $0.40 \pm 0.16$ & 0.14
\end{tabular}

Table 3. Mean (and SE) distribution of critical situational behavior and coping style scores based on different family income levels.

\begin{tabular}{|c|c|c|c|c|c|}
\hline Factor & 1 high & 2 medium & 3 low & $\mathrm{F}$ & LSD \\
\hline & $\mathrm{N}=16$ & $\mathrm{~N}=729$ & $\mathrm{~N}=387$ & & \\
\hline CSB & $19.13 \pm 6.13$ & $15.55 \pm 4.92$ & $16.02 \pm 5.46$ & $4.54^{\star}$ & $1>2,1>3$ \\
\hline PS & $0.68 \pm 0.18$ & $0.76 \pm 0.17$ & $0.74 \pm 0.17$ & 2.88 & \\
\hline SB & $0.47 \pm 0.24$ & $0.37 \pm 0.20$ & $0.41 \pm 0.22$ & $5.70^{\star *}$ & $2<3$ \\
\hline Help & $0.63 \pm 0.22$ & $0.71 \pm 1.99$ & $0.66 \pm 0.21$ & $6.48^{\star *}$ & $2>3$ \\
\hline Fantasy & $0.44 \pm 0.19$ & $0.45 \pm 0.20$ & $0.46 \pm 0.20$ & 0.25 & \\
\hline Retreat & $0.44 \pm 0.12$ & $0.45 \pm 0.17$ & $0.47 \pm 0.17$ & 0.75 & \\
\hline RL & $0.46 \pm 0.13$ & $0.40 \pm 0.17$ & $0.41 \pm 0.17$ & 1.86 & \\
\hline
\end{tabular}


processing power is. Solve problems, seek help, fantasy, rationalization was positively correlated with the ability to cope with critical situations and the ability of solve problems has the highest relevant with the ability to cope with critical situations. And self-blame has the opposite correlation with the ability to cope with critical situations. The data is showed in Table 4.

\subsection{University Students Cope with the Predictive Power of the Capacity to Crisis Situations}

Using multiple linear regressions to explore the six factors of coping styles how to impact of the critical situational behavior. The results showed that factor of self-blame, problem-solving, fantasy, rationalization factors entered into the regression model, a significant role in the prediction of critical situational capacity, with a total explained variance of $21.6 \%$ of the variance. The data is showed in Table 5.

\section{Discussion}

The current study shows the relationship between critical situational behavior and coping styles in college students. We would discussion the current status in college students from five aspects.

\subsection{From the Gender Aspects}

Relative to boys, girls use help coping style more, which is consistent with previous related results (Zhang \& Che, 2005; Yu, 2008). This may the results of different social expect about boys and girls in china. The traditional male roles in China are that male are self-reliance, self-confidence, and decisively, So when facing pressure, men rarely express weakness, lack of self-confidence, Even that one man looking for help means weak ability, which make self-esteem threatened. Consequently, this coping style is less used. However, girls are more vulnerable when they encountered critical situational, so they must seek for the help of others to solve the problem.

Relative to rationalization coping style, boys use it more than girls, which is

Table 4. The relationship of critical situational behavior and coping styles.

\begin{tabular}{ccccccc}
\hline & PS & SB & Help & fantasy & retreat & RL \\
\hline CSB & $-0.346^{* *}$ & $0.349^{* *}$ & $-0.115^{* *}$ & $-0.299^{* *}$ & $0.220^{* *}$ & $-0.247^{* *}$ \\
\hline
\end{tabular}

Table 5. Coping style predicted ability to deal with emergency situations.

\begin{tabular}{ccccc}
\hline CSB & B & Beta & $\mathrm{R}^{2}$ & $\mathrm{t}$ \\
\hline SB & 3.693 & -0.15 & 0.121 & $4.666^{* *}$ \\
Help & -7.82 & 0.258 & 0.179 & $-9.192^{* *}$ \\
Fantasy & 3.813 & -0.15 & 0.204 & $4.939^{* *}$ \\
RL & 3.671 & -0.119 & 0.216 & $4.163^{* *}$ \\
\hline
\end{tabular}


inconsistent with the results of previous studies (Cen \& Zheng, 2005; Li \& Ma, 2009). The result means that boys are more immature in college students compared with who have graduated. And this result also in particular prompted that college mental health education to concern for boys psychological conditions: Not to go for help does not mean that there is no problem. When boys encounter difficulties and setbacks, they can form a good habit of problems faced if they have timely guidance and help.

\subsection{From the Enrollment Category}

In the face of critical situations, inside enrollment deals with issues significantly stronger than the outside enrollment. It may be different ways of tackling problems between Eastern and Western cultures.

\subsection{From the Point of Urban and Rural Sources}

College students from urban have better processing power than who come from rural, which is different from the results of previous studies. This may because that the students from urban have more sources wide field of vision, knowledge, So they can be better deal with and deal with crisis situations and problems.

\subsection{From One-Child Family or Not Aspect}

When encountering problems, students from one-child family tend to use such a mature coping style, however they also more likely to use the retreat style which is less immature coping styles, which is not the same with the results of previous studies ( $\mathrm{Li} \& \mathrm{Xi}, 2006$ ). This may be due to the children from one-child family have many concerns, and social support systems are more powerful, So they are easy to seek for help when they encounter problems. However, when not supported from others, they would use negative way to solve problems. So the result shows that the college students from only child family lack effective method to solve problem, mental health educators need pay close attention when they encounter problems.

\subsection{From the Family Income}

Students from medium and low family income families have stronger ability than who from higher-income families to deal with critical situations. The students from middle-income families are more likely to use help copying style than students from low-income families. college students of low-income families tend to use immature coping style such as self-blame style, indicating that the higher the level of family income, the stronger ability to deal with the problem, and the more likely the use of sophisticated, active coping style. This may be because the lack of strong social support for the poor children of the family economic conditions, prone to inferiority complex, fewer opportunities for social interaction and encounter problems easily remorse. The result prompts colleges and universities to pay special attention to the mental health of the poor stu- 
dents, in addition to economic help should give them spiritual encouragement.

\subsection{Relationship between the Ability of Cope Critical Situational and Copying Style}

The ability of critical situations handling and coping styles in college students exists significantly related, except the factor of self-blame and solve-problem. The four other factors have positive correlation with the ability of cope critical situational. In addition, the factor of self-blame, solve-problem, fantasy and rationalization have good ability to predict the ability of critical situation. That means there is no obvious positive or negative classification in critical behavior and coping style. This means that, for individual ability to deal with critical situations, coping styles exist to ease the individual in the face of the imbalance on the cognitive and behavioral events other than their own ability, so there is no positive and negative. As long as help individuals to reestablish a new equilibrium, are valuable for the individual.

\section{Conclusion}

There were significant differences in the behaviors in emergency situations and the coping style. Meanwhile, the coping styles of self-blame, problem solving, fantasy, rationalization are the distinct predictors for the capacities in emergency situations.

\section{References}

Cen, Y. Y., \& Zheng, X. (2005). The Relative Study between Self-Esteem Level and Coping Styles. Chinese Journal of Clinical Psychology, 13, 167-169.

Dang, X. R., \& Yang, Z. B. (2012). Acute Stress Response Status of the Earthquake Rescue Troops and Its Relationships with Coping Styles. Journal of Shanxi Medical University, 43, 341-344.

Jex, S. M., \& Bliese, P. D. (1999). Efficacy Beliefs as a Moderator of the Effects of Work-Related Stressors: A Multilevel Study. Journal of Applied Psychology, 84, 349-361. https://doi.org/10.1037/0021-9010.84.3.349

Jex, S. M., \& Bliese, P. D. (2001). The Impact of Self-Efficacy on Stressor-Strain Relations: Coping Style as an Explanatory Mechanism. Journal of Applied Psychology, 86, 401-409. https://doi.org/10.1037/0021-9010.86.3.401

Li, R., \& Zhang, Z. Q. (2004). Relationship among Stressful Events, Copying Styles and Mental Health in Medical Students of Military College. Chinese Journal of Clinical Psychology, 12, 165-168.

Li, S. B., \& Xi, B. (2006). The Correlation between Parental Rearing and Coping Style of the College Students from City and Country. Chinese Journal of Behavioral Medical Science, 15, 398-399.

Li, X. N., \& Ma, Q. S. (2009). Coping Style Features of College Students. Health Vocation Education, 17, 22-24.

Liang, B. Y., \& Hao, Z. H. (2005). Development of Chinese College Student Psychological Stress Scale. Studies of Psychology and Behavior, 3, 81-87.

Ning, S., \& Li, C. Y. (2012). Development and Psychometric Analysis of Acute Psycho- 
logical Stress Reaction Questionnaire for Armymen. Journal of Prevent Medical of Chinese People's Liberation Army, 30, 32-35.

Ye, B. J., \& Yang, Q. (2011). Confirmatory Factor Analysis of the Reliability of the Estimated One-Dimensional Test. Educational Measurement and Evaluation, 11, 8-12.

Yu, F. (2008). Psychological Tests. Shanghai: Wenhui Press.

Zhang, L., \& Che, W. B. (2005). A Research on College Students Coping Styles of Psychological Stress. Psychological Science, 28, 36-41. 